\title{
Diversidad, abundancia relativa y patrones de actividad de los mamíferos medianos y grandes, asociados a los bosques riparios del río Bita, Vichada, Colombia
}

Diversity, relative abundance and activity patterns for medium and large mammals of the riparian forests of the Bita River, Vichada, Colombia

\author{
Federico Mosquera-Guerra, Fernando Trujillo, Angélica P. Diaz-Pulido y Hugo \\ Mantilla-Meluk
}

\section{Resumen}

La diversidad de mamíferos en la cuenca del Orinoco es derivada de la mezcla de especies de diferentes regiones biogeográficas y es poco conocida. Por lo tanto se emplearon técnicas de fototrampeo para el registro de los mamíferos medianos y grandes asociados al bosque ribereño en la cuenca alta y media del río Bita, Vichada, Colombia. Se estimó la diversidad, el índice de abundancia relativa y los patrones de actividad. Se instalaron 77 cámaras trampa a lo largo de $200 \mathrm{~km}$ del río en dos periodos hidroclimáticos (baja y alta precipitación). Se obtuvieron en total 16439 imágenes, con un esfuerzo de muestreo de 7700 días-trampa. Se registraron 24 especies de mamíferos, agrupados en 17 familias y 9 órdenes. El índice de diversidad de Shannon-Wiener obtenido a través de este método fue $\mathrm{H}^{\prime}=1,995$, las especies mejor representadas a través del índice de abundancia relativa fueron Tayassu pecari $(\mathrm{IAR}=0,176, \mathrm{n}=1360$ ), Cuniculus paca (IAR = 0,174, $\mathrm{n}=1346$ ), Tapirus terrestris (IAR =0,144, $\mathrm{n}=1114$ ) y Dasyprocta fuliginosa (IAR $=0,116, n=900)$. En términos de patrones de actividad Dasyprocta fuliginosa presentó patrones de actividad diurnos, Leopardus pardalis catemeral, Tayassu pecari crepuscular matutino, Cuniculus paca y Didelphis marsupialis nocturna y Tapirus terrestris crepuscular vespertino. Esta información puede ser de utilidad para la generación de programas para el manejo y conservación de la mastofauna en la cuenca del río Bita.

Palabras claves. Cámaras trampa. Conservación. Escudo Guayanés. Orinoquia colombiana.

\begin{abstract}
The diversity of mammals in the Orinoco River basin is derived from the mixture of species from different biogeographic regions and remains poorly known. Therefore, we used camera trapping techniques for the registration of the large and medium mammals associated to the riparian forest in the upper and middle basins of the Bita River, Vichada, Colombia. Diversity, relative abundance index and activity patterns were estimated. Seventy-seven camera traps were installed along $200 \mathrm{~km}$ of the river in two hydroclimatic periods (low and high precipitation). A total of 16439 images were obtained with a sampling effort of 7700 traps-days. Twenty-four species of mammals were recorded, corresponding to 17 families and 9 orders. The Shannon-Wiener diversity index obtained through this method was
\end{abstract}


$\mathrm{H}^{\prime}=1.995$, the best-represented species through the relative abundance index were Tayassu pecari (IAR $=0.176, \mathrm{n}=1360)$, Cuniculus paca (IAR $=0.174, \mathrm{n}=1346)$, Tapirus terrestris $(\mathrm{IAR}=0.144, \mathrm{n}=1114$ ) and Dasyprocta fuliginosa (IAR $=0.116, \mathrm{n}=900$ ). In terms of activity patterns, Dasyprocta fuliginosa showed a pattern of diurnal activity, Leopardus pardalis catemeral, Tayassu pecari morning twilight, Cuniculus paca and Didelphis marsupialis nocturnal and Tapirus terrestris twilight evening. This information may be useful to plan conservation and management actions for the mastofauna of the Bita River basin.

Keywords. Colombian Orinoquia. Conservation. Guyanese shield. Trap cameras.

\section{Introducción}

La diversidad de mamíferos en la cuenca del Orinoco se encuentra conformada por elementos andinos, guayaneses y amazónicos (Correa et al., 2006). En la cuenca del río Bita se han reportado 63 especies, lo que evidencia una alta diversidad para este grupo taxonómico (Muñoz-Saba et al., 2016; Garrote et al., 2017; Mosquera-Guerra et al., 2017), y representa alrededor del $32,1 \%$ de los mamíferos asociados a los ecosistemas terrestres y acuáticos de la ecorregión de los llanos orientales colombianos (Pardo-Martínez y Rangel-Ch, 2014), $19,8 \%$ de los registrados para la cuenca del río Orinoco (Ferrer et al., 2009) y 12,1 \% de las 518 especies de mamíferos del país (Solari et al., 2013; Ramírez-Chaves y Suárez-Castro, 2014; RamírezChaves et al., 2016).

La mastofauna asociada a los bosques ribereños en la cuenca del río Bita presenta una gran importancia ecológica y funcional (Trujillo y Mosquera-Guerra, 2016; Mosquera-Guerra et al., 2017). En estos ecosistemas habitan especies claves e ingenieras como grandes carnívoros (Panthera onca, Puma concolor y Leopardus pardalis) (Garrote et al., 2017; Mosquera-Guerra et al., 2017), perisodáctilos (Tapirus terrestris), artiodáctilos (Tayassu pecari y Odocoileus cariacou) y xenartros (Priodontes maximus y Myrmecophaga tridactyla) (Muñoz-Saba et al., 2016; Mosquera-Guerra et al., 2017). Su presencia es indicadora de diversos tipos de recursos como agua, alimento y refugio en escalas espaciales y temporales distintas (Trujillo y Mosquera-Guerra, 2016; Mosquera-Guerra et al., 2017).
La abundancia relativa de los mamíferos es un indicador de la situación poblacional y su evaluación en diferentes escalas (espacial y temporal) permite evidenciar posibles variaciones (Wilson et al., 1996; Walker et al., 2000; MonroyVilchis, et al. 2011). Este parámetro, al igual que el patrón de actividad, puede contribuir a la propuesta de estrategias para la conservación de las especies (Walker et al., 2000; Blake et al., 2012). La estimación de la abundancia para el caso de mamíferos tanto medianos como grandes es difícil y costosa debido a que sus hábitos son nocturnos y evasivos, y a que, por lo general, se encuentran en bajas densidades. Por lo anterior, es recomendable el cálculo de índices de abundancia relativa (Sutherland, 1996). Los valores de los índices de abundancia se basan en el resultado del muestreo de una fracción de la población y se expresan como el número de individuos registrados por unidad de muestreo ( $\mathrm{O}^{\prime}$ Brien et al., 2003; Yasuda, 2004; Rovero y Marshall, 2009). Esta información puede ser obtenida a través del uso de cámaras trampa, lo cual permite enfocarse en estudios poblacionales de mamíferos con una herramienta confiable y no invasiva de muestreo (Krausman, 2002; Silveira et al., 2003; Pinto de Sá Alves y Andriolo, 2005; Monroy-Vilchis et al., 2009); además de su efectividad, el uso de cámaras trampa permite la obtención de registros de especies crípticas, que se mueven largas distancias o que presentan bajas densidades (Srbek-Araujo y García, 2005; Monroy-Vilchis et al., 2009, 2011). Adicionalmente, las cámaras trampa confieren precisión en la identificación a nivel taxonómico 
$\mathrm{y}$, frecuentemente, individual. Su eficiencia es similar en la detección de especies con hábitos diurnos y nocturnos (Maffei et al., 2002; Trolle y Kéry, 2003; Monroy-Vilchis et al., 2011).

Esta herramienta es usada para múltiples propósitos, desde identificar individuos de una población hasta evaluar su tamaño (Lira-Torres y Briones-Salas, 2012). En la actualidad se ha incrementado el uso sistematizado de cámaras trampa para estimar el índice de abundancia relativa (IAR), parámetro que se correlaciona con la densidad poblacional (Maffei et al., 2002; MonroyVilchis et al., 2011; Lira-Torres y Briones-Salas, 2012), además de describir patrones de actividad (Maffei et al., 2002; Pinto de Sá Alvez y Andriolo, 2005; Monroy-Vilchis et al., 2009), establecer el uso del hábitat (Bowkett et al., 2007; Monroy-Vilchis et al., 2009; Lira-Torres y Briones-Salas, 2011), y estimar aspectos etológicos de las especies (Maffei et al., 2005; Trolle y Kéry, 2005; Haines et al., 2006).

Los patrones de actividad de muchos mamíferos en el Neotrópico se conocen en términos generales (es decir, si una especie es principalmente nocturna o diurna) (por ejemplo, Emmons y Feer, 1997), pero los detalles de estos patrones están menos estudiados (Blake et al., 2012). Por esta razón, es necesario generar información acerca de los patrones de actividad de los mamíferos tropicales en aras de aumentar nuestra comprensión básica de su ecología (competencia y depredación) y potencialmente, para entender los impactos de las actividades humanas sobre el comportamiento de las especies afectadas (por ejemplo, las que son cazadas) (Blake et al., 2012).

En consecuencia, el fototrampeo es una de las metodologías más relevantes y versátiles para la investigación biológica con fines de conservación (Trolle y Kéry, 2003; Maffei et al., 2004; Lira-Torres y Briones-Salas, 2012). Por lo anterior, los objetivos de la presente investigación fueron determinar la diversidad, el índice de abundancia relativa y los patrones de actividad de los medianos y grandes mamíferos asociados a los bosques riparios en la cuenca media y alta del río Bita, Vichada,
Colombia, con el fin de proporcionar insumos para el manejo y conservación de esta cuenca.

\section{Materiales y métodos}

Área de estudio. Se localiza en la cuenca alta y media del río Bita, en los municipios de la Primavera y Puerto Carreño, Vichada, Colombia, a una altitud entre 45 y 103 m s.n.m, entre las coordenadas $5^{\circ} 51^{\prime}$ a $5^{\circ} 43^{\prime} \mathrm{N}$ y $69^{\circ} 4^{\prime}$ a $68^{\circ} 10^{\prime} \mathrm{O}$ (Mosquera-Guerra et al., 2017) (Figura 1). La temperatura varía dependiendo de la estación climática, entre los meses de enero a abril durante el periodo de bajas precipitaciones oscila entre los $35^{\circ}$ y $43{ }^{\circ} \mathrm{C}$ y en altas precipitaciones correspondiente a los meses de mayo a septiembre entre los $25^{\circ}$ y $35^{\circ} \mathrm{C}$ (Corporinoquia et al., 2015). La vegetación dominante en los helobiomas son conocidos comúnmente como bosques ribereños, riparios o de galería, y se encuentran ubicados sobre las márgenes de los grandes ríos, y pequeños tributarios de toda la cuenca del Orinoco (Hernández Camacho, 1997; Romero et al., 2004).

\section{Registro de mamíferos medianos y grandes.} El muestreo se desarrolló entre los meses de diciembre de 2015 y mayo de 2016, con un promedio de 50 días de muestreo para cada periodo hidroclimático evaluado, correspondiente a periodos de bajas (diciembre-enero) y altas (mayo-junio) precipitaciones, cubriendo un total de 100 días.

Se establecieron 77 estaciones de fototrampeo que fueron georreferenciadas con un geoposicionador (GPSMAP® 62sc | Garmin). En el primer periodo de muestreo (bajas precipitaciones), se instalaron 37 cámaras trampa y las 40 restantes durante el periodo (altas precipitaciones), los equipos de fototrampeo empleados fueron de referencia Bushnell Trophy Cam. Se ubicaron a una altura entre 40 y $50 \mathrm{~cm}$ del nivel del suelo, con una separación aproximada de $1 \mathrm{~km}$ de distancia entre cada una, cubriendo un longitud de $200 \mathrm{~km}$, ubicadas de manera paralela al río y asociadas a la vegetación ribereña. El circuito de la cámara- 


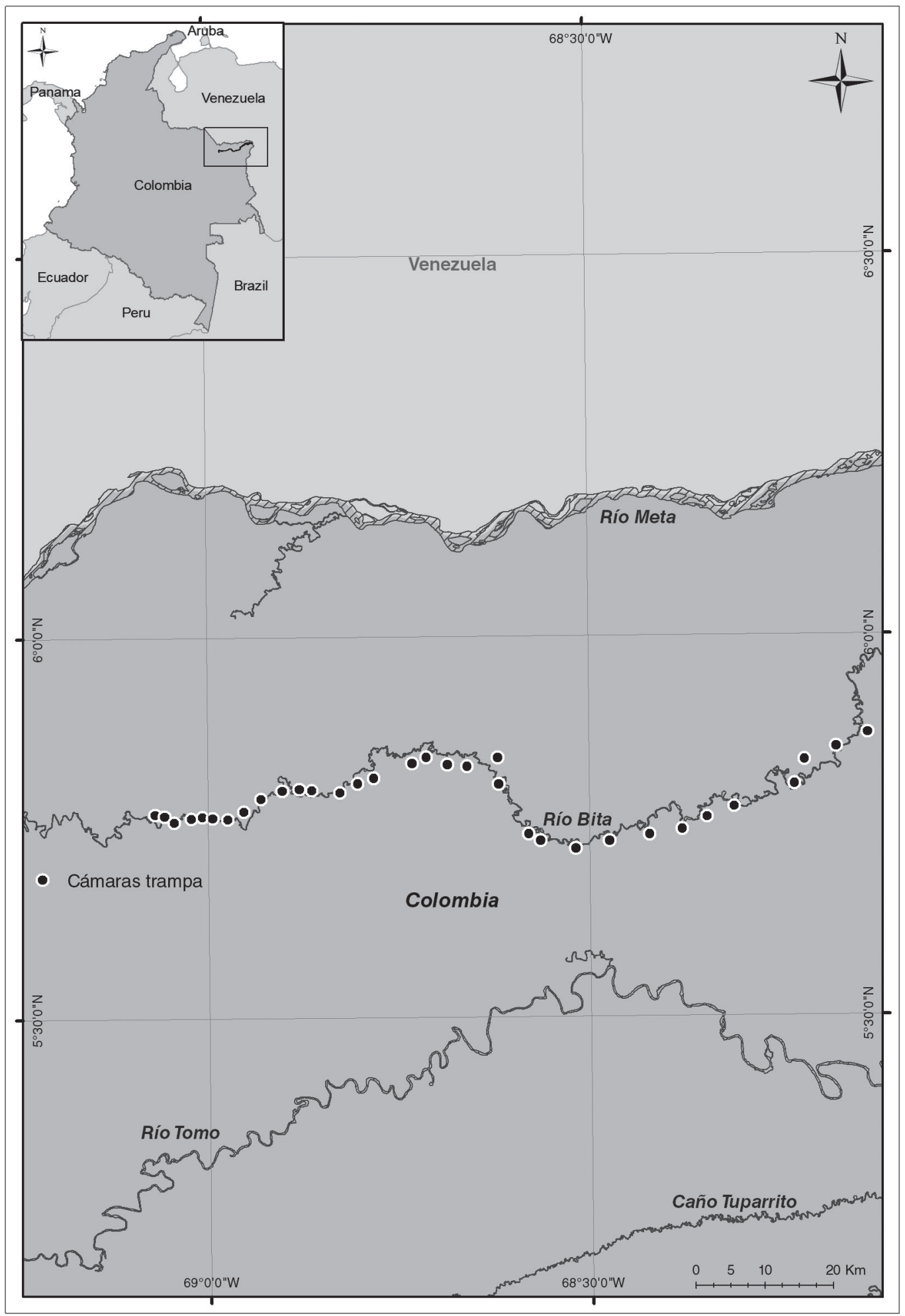

Figura 1. Ubicación de las cámaras trampa en la cuenca alta y media del río Bita, Vichada, Colombia. 
trampa fue programado para permanecer activo durante 24 horas y fueron programadas para registrar 3 fotografías y 1 video de 20 segundos de duración a partir del estímulo activador a un intervalo de 1 segundo entre la primera y segunda ráfaga o hasta que apareciera un nuevo objetivo, en cada fotografía se registró la fecha, hora, fase lunar y temperatura $\left({ }^{\circ} \mathrm{C}\right)$.

Análisis de la información. El esfuerzo de muestreo se obtuvo multiplicando el número total de cámaras-trampa por el total de días que permanecieron activas (Medellín et al., 2006). Los individuos registrados fueron identificados siguiendo la literatura especializada (Emmons y Feer, 1997; Morales-Jiménez et al., 2004; Wilson y Reeder, 2005). La representatividad del muestreo se determinó a través de los estimadores de riqueza $\mathrm{ACE}$, Chao 1 y Cole, que utiliza los datos de abundancia y se basa en el número de especies raras en la muestra (Gotelli y Colwell, 2001). Los cálculos se llevaron a cabo utilizando el software EstimateS ver. 8.2.0 (Colwell, 2006).

El gremio trófico de las especies registradas se clasificó en herbívoros, insectívoros, frugívoros, carnívoros y omnívoros, de acuerdo con MuñozSaba et al. (2016). Finalmente, para conocer la condición de amenaza de los mamíferos registrados se consultó el libro rojo de mamíferos de Colombia (Rodríguez-Mahecha et al., 2006) y la lista roja de la Unión Internacional para la Conservación de la Naturaleza (UICN, 2016).

La diversidad alfa (a) se estimó para cada método, de acuerdo con el índice de Shannon-Wiener. Para las temporadas de bajas y altas precipitaciones, el índice se comparó por medio de la prueba $t$ modificada de Hutchinson (Moreno, 2001). Además, se estimó la dominancia a través del índice de Berger-Parker (Whittaker, 1972), que considera la importancia proporcional de las especies más abundantes y, el índice de equidad de Pielou (Magurran, 1988), que mide la proporción de la diversidad observada con relación a la máxima diversidad esperada (Moreno, 2001).
El índice de abundancia relativa de las especies se determinó a través del número de registros fotográficos independientes con relación al esfuerzo de muestreo. Un registro independiente considera: fotografías consecutivas de diferentes individuos, fotografías consecutivas de individuos de la misma especie separadas por más de 24 horas y fotografías no consecutivas de individuos de la misma especie. Para las especies gregarias, el número de registros independientes fue igual al número de individuos observados en una fotografía (Medellín et al., 2006; Monroy-Vilchis et al., 2011; Lira-Torres y Briones-Salas, 2012). Se calculó la abundancia relativa por temporada y se realizó una prueba de Mann-Whitney para determinar diferencias significativas entre temporadas (Zar, 1999).

Se determinó el patrón de actividad general y por temporada para aquellas especies que contaran con el mayor número de registros independientes (Maffei et al., 2002; Monroy-Vilchis et al., 2011). Las imágenes obtenidas se agruparon en intervalos de 2 horas y el patrón de actividad se clasificó en diurnas (de las 8:00 a las 18:00 horas), nocturnas (de las 20:00 a las 06:00 horas), crepuscular (matutino, entre las 6:00 y las 8:00 horas y vespertino entre las 18:00 y las 20:00 horas), las especies que no mostraron un patrón claro se clasificaron como catemerales (Maffei et al., 2002; Monroy-Vilchis et al., 2011).

\section{Resultados y discusión}

Las metodologías tradicionales empleadas para la realización de investigaciones en aspectos poblaciones como tamaños, índices de abundancia y densidad para medianos y grandes mamíferos como: recorridos a lo largo de transectos, conteos directos y técnicas de marca-recaptura, requieren de mucho esfuerzo y están muy limitados por las condiciones ambientales presentes donde habitan estas especies (Gaidet-Drapier et al., 2006; Roberts et al., 2006; Swan et al., 2014). Para la realización del presente estudio, dada la escala y la geomorfología de la cuenca, se implementó 
el uso de cámaras trampa debido al grado de precisión en la obtención de los registros para este grupo taxonómico (Cervantes y Yépez, 1995), estableciendo a través de este método de muestreo la presencia de 24 especies correspondientes a 13 familias de 7 órdenes, el $38 \%$ de los mamíferos medianos y grandes reportados para la cuenca del Bita (Muñoz-Saba et al., 2016; Garrote et al., 2017; Mosquera-Guerra et al., 2017) (Anexo 1).

El orden mejor representado fue Carnivora, con 4 familias y 9 especies, durante el periodo seco se registraron 24 especies en 2583 registros independientes, mientras que durante la temporada de lluvias se detectaron 17 especies en 3244 registros independientes (Tabla 1). Los estimadores de riqueza $\mathrm{ACE}$, Chao 1 y Cole, determinaron que el número de especies observadas estuvieron representadas entre un $90 \%$ y $95 \%$ de las especies esperadas. Estos resultados coinciden con lo expuesto por Monroy-Vilchis et al. (2011) y Lira-Torres et al. (2012) y valores que permiten establecer un elevado nivel de eficacia y representatividad para los muestreos realizados a través de este método no invasivo en los dos periodos hidrológicos evaluados.

El fototrampeo obtuvo además de los registros de los especímenes, información relevante acerca de diversos aspectos ecológicos como abundancia relativa, patrón de actividad e incluso información que puede ser útil para hacer inferencias acerca de aspectos reproductivos; como por ejemplo los registros de hembras con crías para Lontra longicaudis, Tapirus terrestris y Dasyprocta fuliginosa, así como parejas de hembras y machos adultos para Tayassu pecari e Hydrochoerus hydrochaeris (Monroy-Vilchis et al., 2009, 2011; Lira-Torres et al., 2012) (Figura 2).

De los 24 mamíferos registrados, los gremios tróficos estuvieron representados por los omnívoros ( $n=9 ; 37,5 \%)$, carnívoros $(n=6 ; 25,0 \%)$, herbívoros/frugívoros $(\mathrm{n}=5 ; 20,8 \%)$, insectívoros ( $\mathrm{n}=3 ; 12,5 \%$ ), y 1 especie fue herbívora. Estos resultados coinciden con lo expuesto por Muñoz Saba et al. (2016) para la cuenca del río Meta, por Trujillo y Mosquera-Guerra (2016) para los ecosistemas de morichales de la Orinoquia y por Mosquera-Guerra et al. (2017) para el río Bita. Se reportan: (A) depredadores tope como los felinos y mustélidos (carnívoros); (B) herbívoros/ frugívoros (artiodáctilos, perisodáctilos y roedores), que contribuyen, de forma directa o indirecta, al mantenimiento o regeneración de los bosques porque consumen frutos, semillas y polen, por lo tanto favorecen los procesos de dispersión de semillas (Cadena et al., 1998); y (C) armadillos y osos hormigueros (insectívoros), que contribuyen en el control de las poblaciones
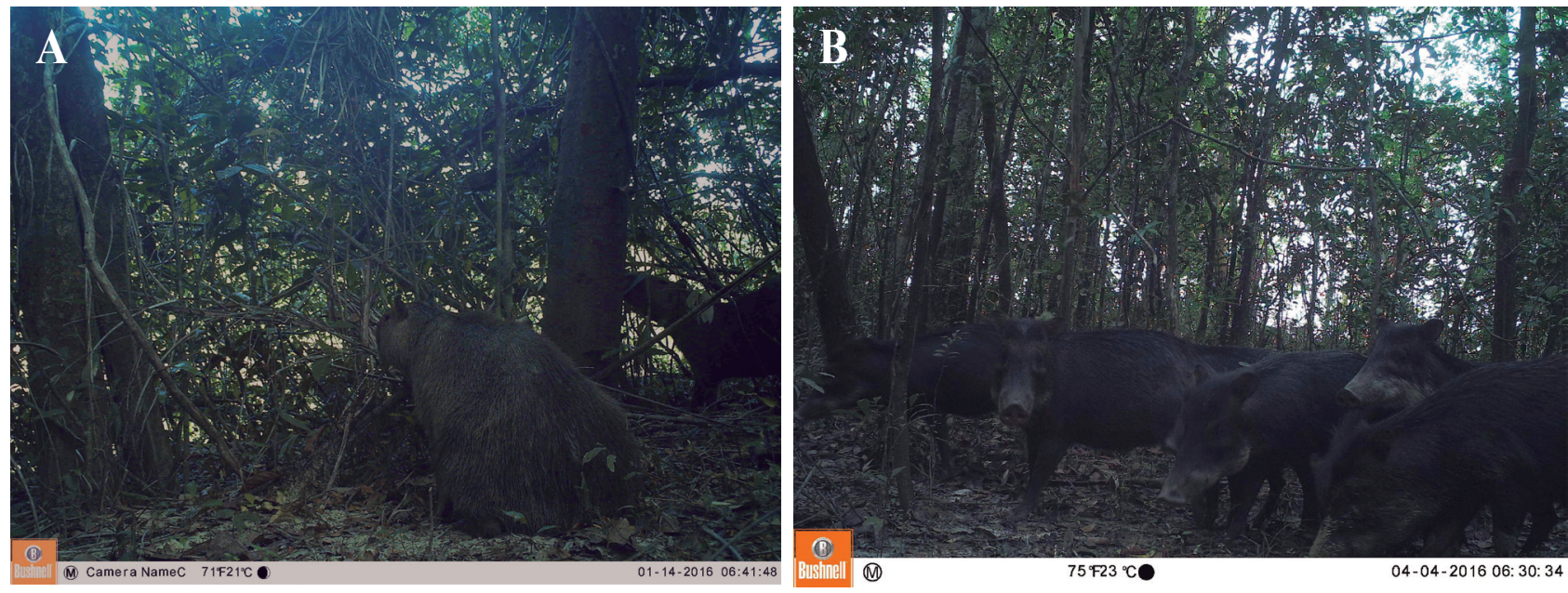

Figura 2. Presencia de parejas de hembras y machos adultos para A) Hydrochoerus hydrochaeris y B) Tayassu pecari en los bosques riparios del río Bita, Vichada, Colombia. 
de insectos que pueden convertirse en plagas o vectores de enfermedades (Naranjo y Amaya, 2009).

La diversidad de mamíferos medianos y grandes estimada a través del índice de Shannon-Wiener registrados con cámaras trampa fue de $\mathrm{H}^{\prime}=$ 1,995, con valores para la diversidad máxima de $\mathrm{H}^{\prime} \max =2,02$. El valor del índice de diversidad fue significativamente mayor durante el periodo seco $(p=0,02)$. Los valores obtenidos para el índice Berger-Parker evidencian un grado de dominancia bajo para la comunidad de mamíferos a lo largo de los periodos evaluados $(\mathrm{D}=0,17)$, para las temporada seca $(\mathrm{D}=0,20)$ y lluvias $(\mathrm{D}=$ $0,22)$. El valor en el índice de equidad de Pielou correspondió a J = 0,62 durante todo el estudio, y para la temporada lluviosa fue un poco más alto que para la temporada seca (Tabla 1).

Las especies que presentaron los mayores valores en el índice de abundancia relativa fueron Tayassu pecari (IAR $=0,176, \mathrm{n}=1360$ ), Cuniculus paca (IAR $=0,174, \mathrm{n}=1346$ ), Tapirus terrestris (IAR $=0,144, \mathrm{n}$ $=1114)$, Dasyprocta fuliginosa $(\mathrm{IAR}=0,116, \mathrm{n}=900)$; mientras que Coendou prehensilis (IAR $=0,0002, \mathrm{n}$ = 2), Procyon cancrivorus y Dasypus kappleri (IAR =
$0,0001, \mathrm{n}=1$ ) fueron las menos abundantes. Las especies más abundantes durante los periodos evaluados correspondieron a la danta de tierra bajas (Tapirus terrestris) en el periodo seco (IAR $=$ $0,05, \mathrm{n}=729)$ y Tayassu pecari $(\mathrm{IAR}=0,15, \mathrm{n}=1360)$ en lluvias. En relación a los valores del índice de abundancia entre los periodos hidrológicos evaluados, no se observaron diferencias significativas $(\mathrm{U}=242, p=0,34)$.

En la actualidad se discute la veracidad de emplear los valores de la frecuencia de captura como un índice de abundancia relativa y algunos autores han argumentado que el número de fotografías obtenidas de una especie depende de la probabilidad de detección, más que de la abundancia de la misma (Tobler et al., 2008; Harmsen et al., 2010; Monroy-Vilchis et al., 2011). Sin embargo, autores como Kelly y Holub (2008) y Rowcliffe y Carbone (2008), establecen que la consistencia que generalmente se observa entre los valores para los índices obtenidos en diferentes periodos evaluados, puede aumentar la confiabilidad de la frecuencia de captura para estimar la abundancia relativa de las especies controlando algunas variables (tipos de vegetación, periodos hidroclimáticos y senderos empleados

Tabla 1. Índices ecológicos calculados para la comunidad de mamíferos registrados en la cuenca alta y media del río Bita, Vichada, Colombia.

\begin{tabular}{lccc}
\hline & \multicolumn{3}{c}{ Fototrampeo } \\
\cline { 2 - 4 } & Temporada seca & Temporada de lluvias & Total \\
\hline Esfuerzo de muestreo & 3850 & 3850 & 7700 \\
\hline Registros independientes & 2583 & 3244 & 5827 \\
\hline Especies registradas & 24 & 17 & 24 \\
\hline Especies exclusivas & 7 & 0 & 7 \\
\hline Diversidad Shannon-Wiener $\left(\mathrm{H}^{\prime}\right)$ & 1,975 & 1,802 & 1,995 \\
\hline H'max & 2,018 & 1,836 & 2,02 \\
\hline Dominancia $(\mathrm{D})$ & 0,206 & 0,223 & 0,174 \\
\hline Equidad $\left(\mathrm{J}^{\prime}\right)$ & 0,621 & 0,636 & 0,627 \\
\hline
\end{tabular}


por los mamíferos). Estos podrían llegar a influir positivamente en los índices de captura, de esta forma es posible extraer información como la abundancia basada en frecuencias de fototrampeo (Monroy-Vilchis et al., 2011). En relación a la alteración en los patrones etológicos de las especies por el uso de esta técnica, esta no puede ser considerada como una desventaja debido a que los resultados expuestos han sido contradictorios; Monroy-Vilchis et al. (2011), expone un ejemplo de esta contradicción a través de los resultados obtenidos Gompper et al. (2006), sugiriendo que los coyotes evitan las trampas-cámara. Sin embargo, Kelly y Holub (2008) y Pina et al. (2004) reportan que en el estudio que realizaron en Virginia esta especie fue una de las que presentó un mayor éxito de captura por las cámaras trampas. De acuerdo con los IAR calculados, las especies más abundantes fueron Tayassu pecari, Tapirus terrestris, Cuniculus paca y Dasyprocta fuliginosa, lo cual coincide con lo observado en los recorridos realizados por Mosquera-Guerra et al. (2017) con respecto al encuentro de evidencia como rastros, heces y comederos.

Entre las especies con mayor número de registros, Dasyprocta fuliginosa (04:00-8:00 hrs) presentó patrones de actividad diurnos coincidiendo con lo expuesto por Blake et al. (2012), Leopardus pardalis (8:00-12:00 hrs) catemeral (Emmons, 1988), Tayassu pecari (04:00-9:00 hrs) crepuscular matutino (Blake et al., 2012), Cuniculus paca (02:0006:00 hrs) nocturna, Didelphis marsupialis (00:003:00 hrs) nocturna y Tapirus terrestris (18:0022:00 hrs) crepuscular vespertino (Blake et al., 2012). Solo se evidenció un cambio en el patrón de actividad diurno a nocturno para Leopardus pardalis (19:00-1:00 hrs) durante el periodo de alta precipitación, esta modificación en la actividad de los depredadores puede variar geográficamente para igualar la actividad de las presas potenciales (Maffei et al., 2004; Weckel et al, 2006) (Figura 3, Tabla 2). Las especies registradas exclusivamente en la temporada seca, correspondieron a Lontra longicaudis, Pteronura brasiliensis, Nasua nasua, Procyon cancrivorus, Dasypus kappleri, Hydrochoerus hydrochaeris y Coendou prehensilis.

En relación a los análisis de los patrones de actividad, Van Schaik y Griffiths (1996), exponen la correlación entre el tamaño corporal de los animales más grandes (dantas, chácharos y venados) y sus requerimientos energéticos más elevados, ocasionando la necesidad de forrajear durante periodos prolongados de tiempo, por lo que son activos tanto en el día como en la noche; mientras que los mamíferos que presentan hábitos nocturnos corresponden a animales pequeños $(<10 \mathrm{~kg})$ y están relacionados con comportamientos de evasión debido al riesgo de depredación. Sin embargo, algunos de los resultados de la presente investigación son contradictorios con esta aseveración, debido a que especies de menos de $10 \mathrm{~kg}$, como el ñeque (Dasyprocta fuliginosa) fueron más activos en horas del día principalmente, por lo que es más probable que factores externos (temperatura, humedad, disponibilidad de recursos, etc.), comportamientos inherentes a los individuos de cada especie (sexo, edad, estatus reproductivo, etc.), además de evitar la competencia con especies relacionadas o ecológicamente similares, como Cuniculus paca (Tobler et al., 2009), tengan mayor influencia sobre el patrón de actividad (Monroy-Vilchis et al., 2011; Blake et al., 2012). Esta alteración también puede presentarse en especies muy presionadas por actividades de cacería, debido a esta actividad tienden a presentar comportamientos nocturnos como una estrategia antidepredación, esto correspondería con la información registrada para Cuniculus paca y Tapirus terrestris que presentaron una mayor actividad nocturna (Blake et al., 2012; Mosquera-Guerra et al., 2017). Los patrones de actividad para Leopardus pardalis durante el periodo de lluvias corresponde con lo registrado por Ayala et al. (2010) y Díaz-Pulido y PayánGarrido (2011), ya que esta especie se ha reportado con actividad exclusivamente nocturna. 
Cuniculus paca

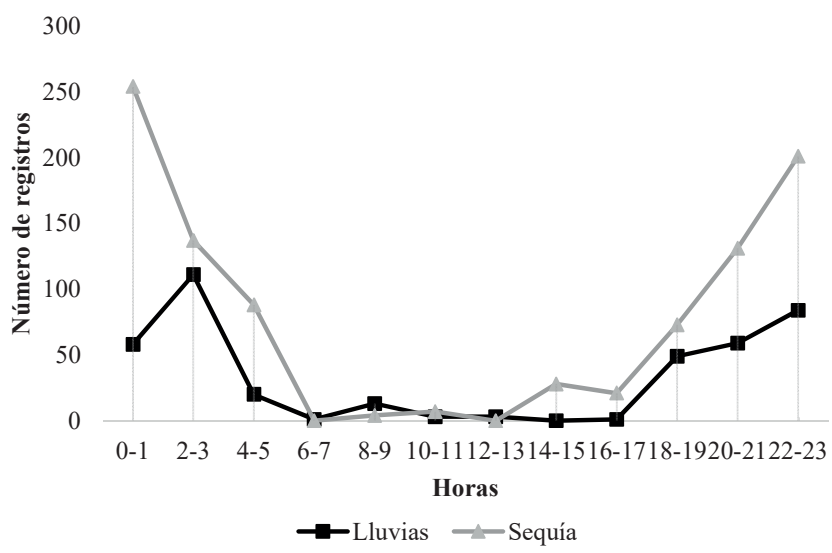

Didelphis marsupialis
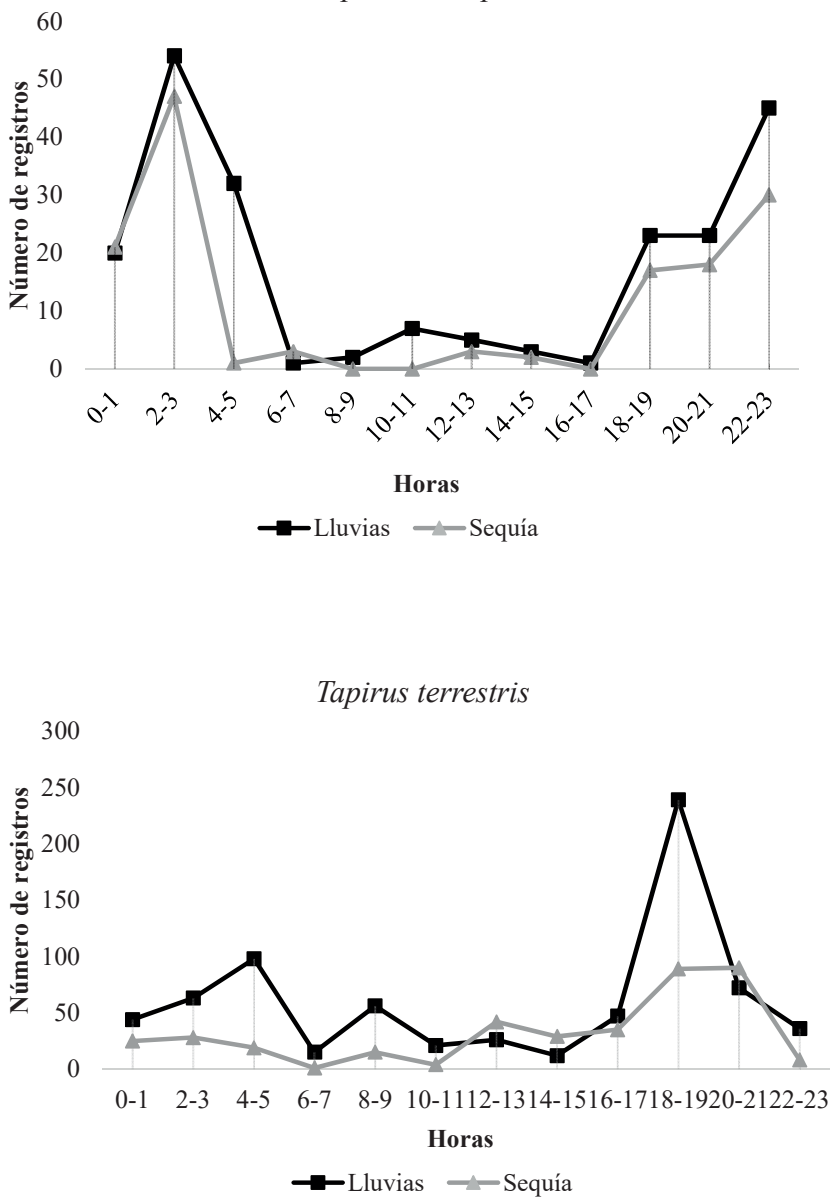

Dasyprocta fuliginosa
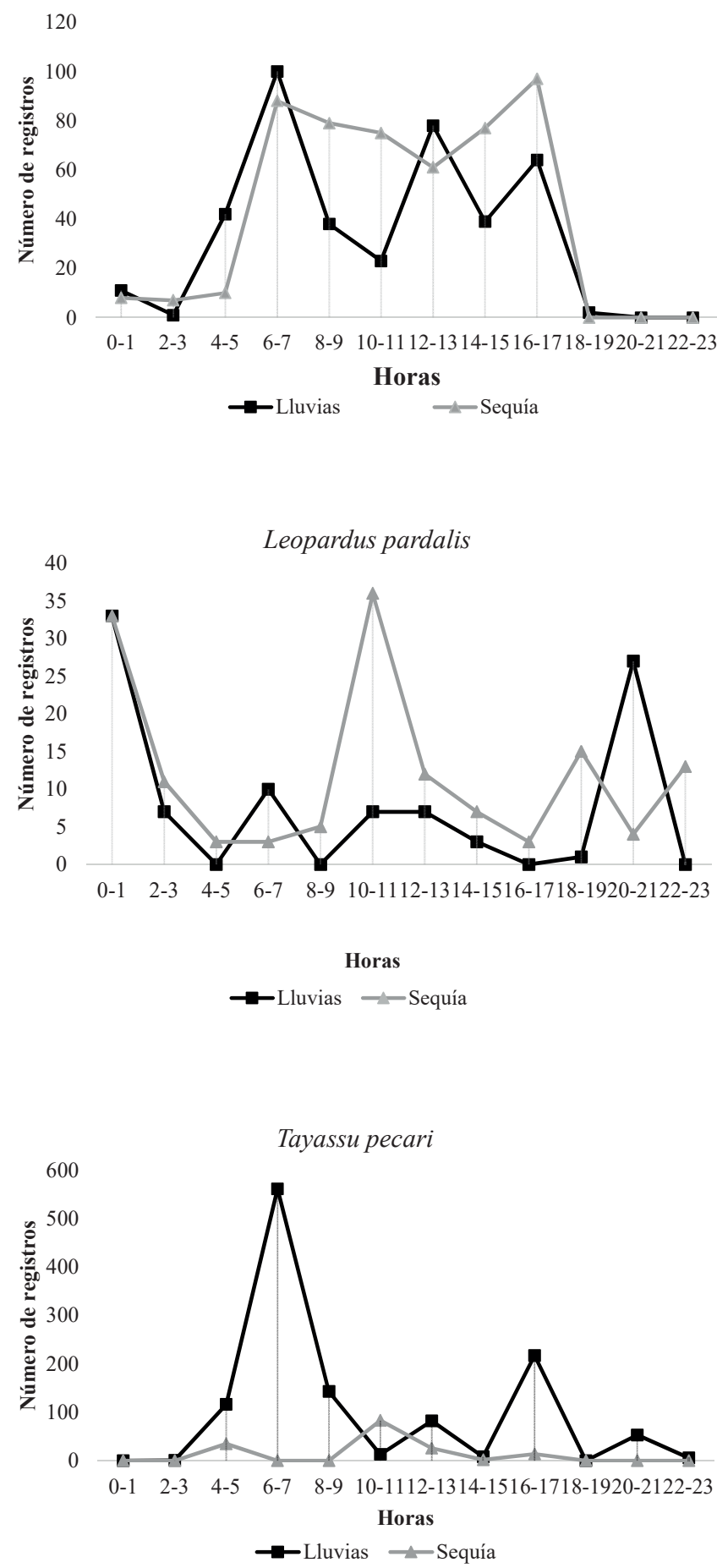

Figura 3. Patrones de actividad de los mamíferos medianos y grandes más abundantes en la cuenca alta y media del río Bita, Vichada, Colombia. 


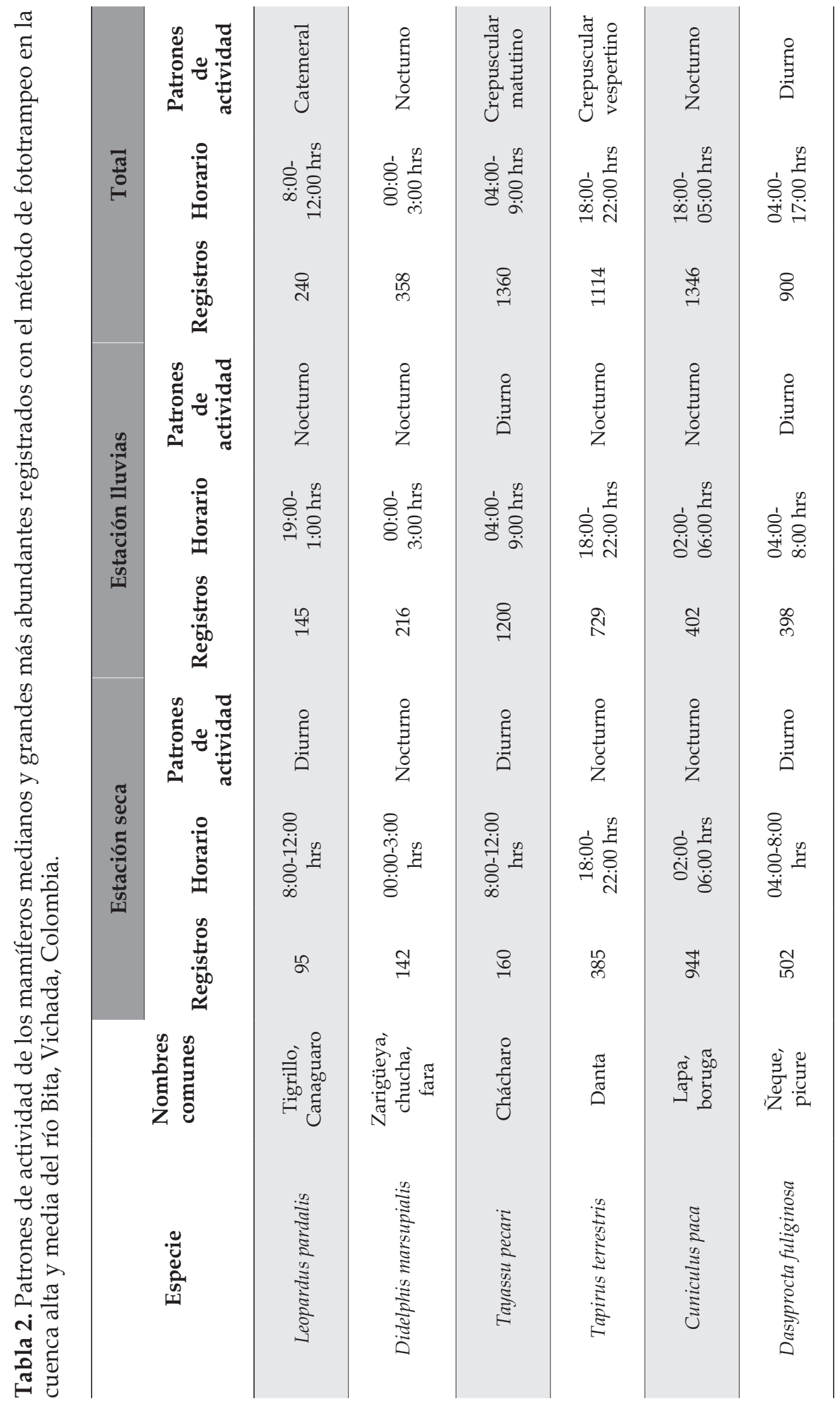

Biota Colombiana 19 (1) - 2018211 
El 16,6 \% de las especies registradas se encuentran bajo alguna categoría de amenaza. Son reportadas cuatro especies por RodríguezMahecha et al. (2006): Lontra longicaudis (VU), Pteronura brasiliensis (EN), Tapirus terrestris (CR) y Myrmecophaga tridactyla (VU). Tayassu pecari se encuentra dentro de la lista internacional (UICN, 2016) como Vulnerable (Figuras 4a y 4b). La presencia de carnívoros como Puma concolor, Leopardus pardalis y Herpailurus yagouaroundi, sugiere que el bosque ribereño en la cuenca alta y media del río Bita se encuentra en buen estado de conservación, ya que al ser estas especies los depredadores tope necesitan amplios requerimientos de hábitat para poder desarrollarse y tener una viabilidad poblacional (Holden y Neang, 2009; Cueva et al., 2010). Sin embargo en los muestreos realizados empleando cámaras trampa no se ha logrado registrar el jaguar (Panthera onca), posiblemente su distribución se asocia más a coberturas forestales dominadas por los morichales cerca a las sabanas donde depreda animales domésticos (Bos indicus y Sus scrofa), agudizando así los conflictos entre ganaderos y grandes carnívoros (Garrote et al., 2017).
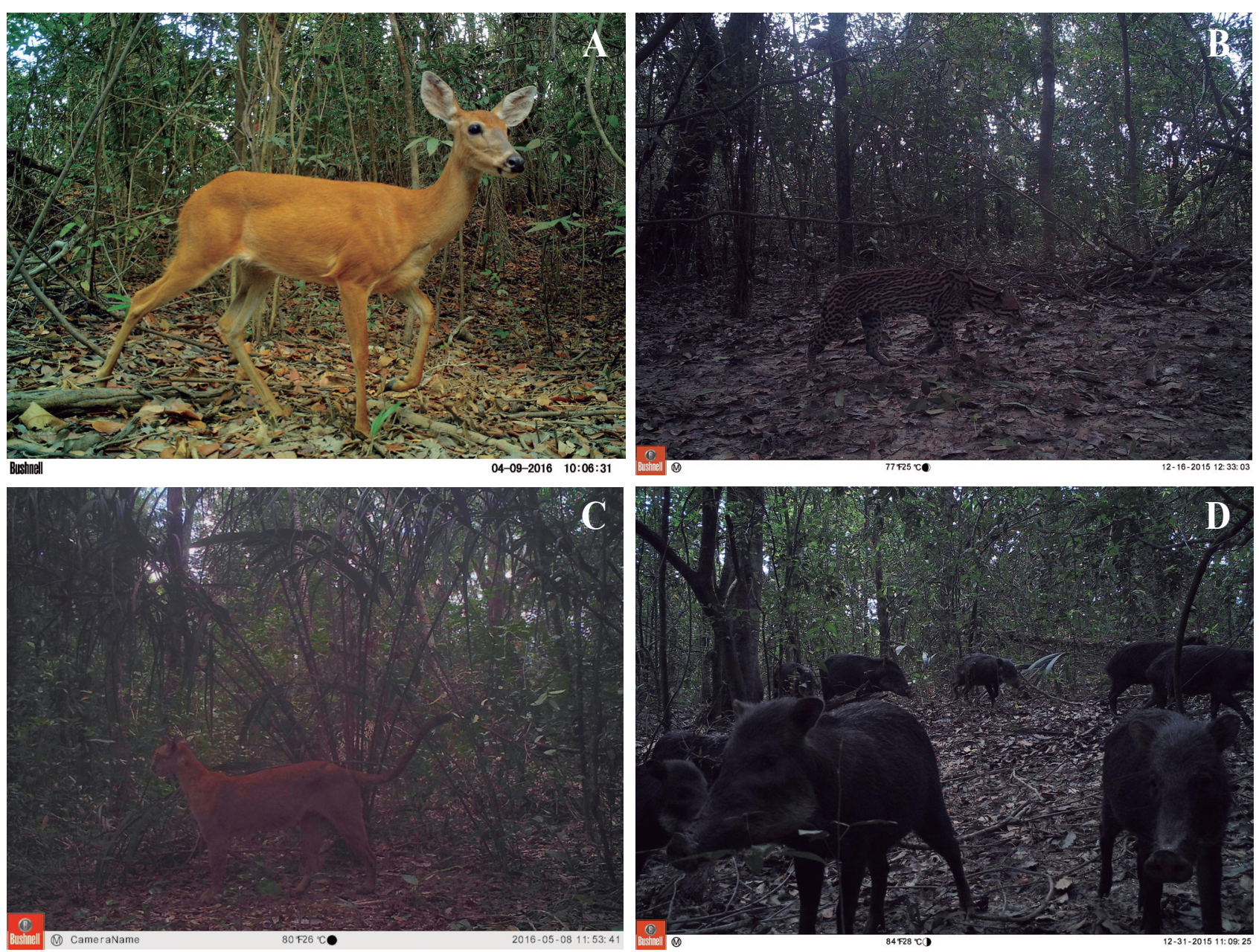

Figura 4a. Mamíferos registrados por cámaras trampa en la cuenca alta y media del río Bita, Vichada, Colombia. A) Odocoileus cariacou, B) Leopardus pardalis, C) Puma concolor y D) Tayassu pecari. 

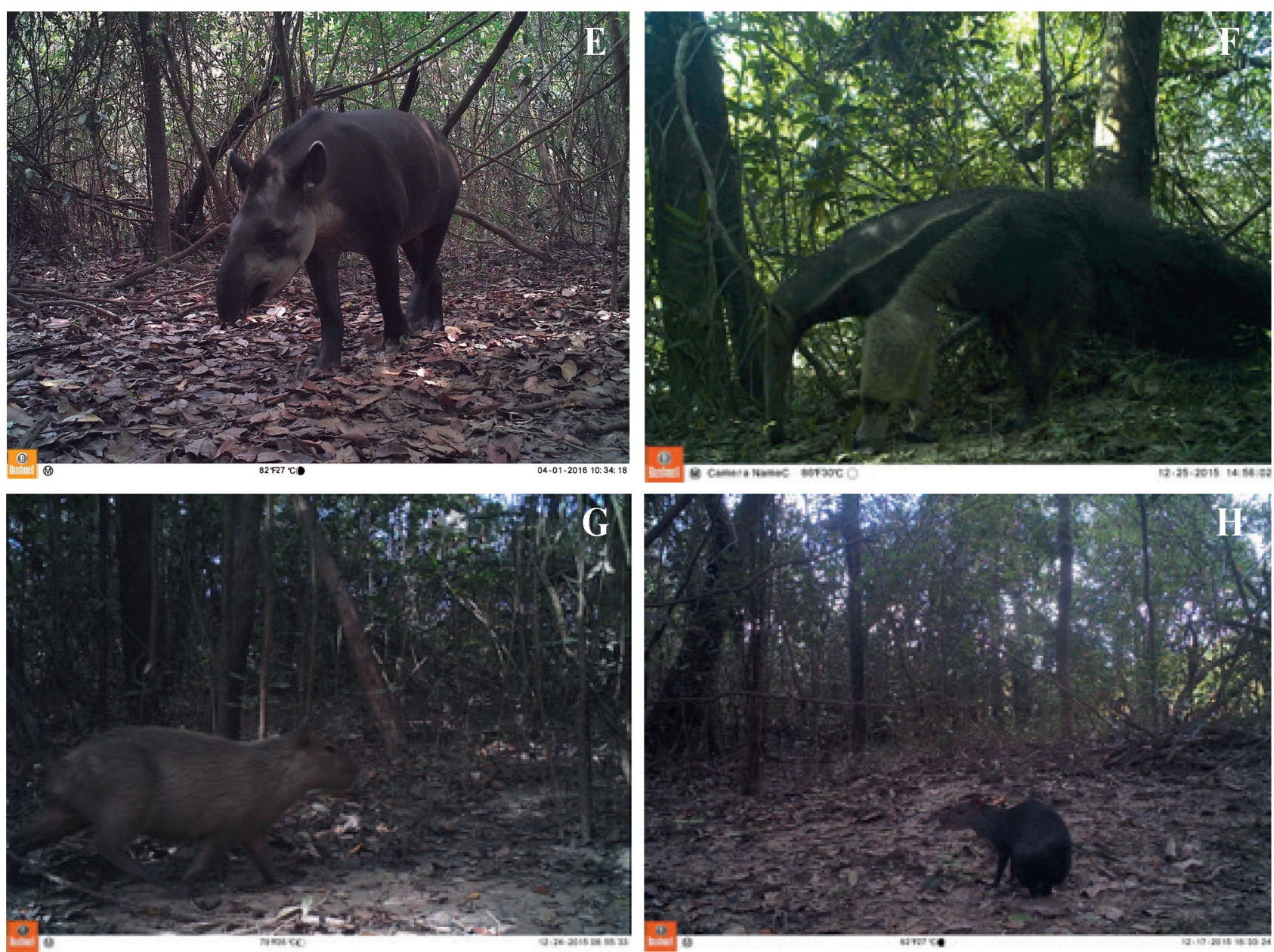

Figura 4b. Mamíferos registrados por cámaras trampa en la cuenca alta y media del río Bita, Vichada, Colombia. E) Tapirus terrestris, F) Myrmecophaga tridactyla, G) Hydrochoerus hydrochaeris y H) Dasyprocta fuliginosa.

\section{Conclusiones y recomendaciones}

El uso de cámaras trampa permitió registrar para la cuenca alta y media del río Bita el 7,6 \% de los mamíferos medianos y grandes de Colombia (Ramírez-Chávez et al., 2016) y el 14,2 \% de los reportados para la Orinoquia (Ferrer et al., 2009), evidenciando la efectividad de esta técnica en el registro de especies cripticas y evasivas como los ungulados (Tapirus terrestris, Odocoileus cariacou y Tayassu pecari) y felinos (Puma concolor, Leopardus pardalis y Herpailurus yagouaroundi) que presentan problemas de conservación por cacería, transformación de sus hábitats y eventos de muerte por retaliación como el caso de los grandes carnívoros.

Los registros obtenidos a través de cámaras trampa de especies claves e ingenieras como los grandes carnívoros, perisodáctilos y artiodáctilos, evidencian la importancia de las coberturas forestales asociadas al río Bita y su papel como corredor biológico para los medianos y grandes mamíferos que se desplazan entre los ecosistemas 
del Escudo Guayanés y las sabanas inundables de la cuenca del Orinoco (Trujillo y MosqueraGuerra et al., 2016; Mosquera-Guerra et al., 2017).

Se recomienda establecer un plan de monitoreo para los mamíferos medianos y grandes empleando técnicas de fototrampeo a largo plazo en aras de evaluar estacionalmente la presencia y abundancia de estas especies, al igual que realizar estudios de composición florística de los ecosistemas forestales asociados a estas coberturas para ver los aportes en la dieta de especies frugívoras y herbívoras como los grandes ungulados.

\section{Agradecimientos}

Los autores quieren expresar su agradecimiento a los investigador local Jacinto Teherán de la Fundación Omacha, propietarios y encargados de los predios Anakai, Mi Familia, La Florida y Rampla Vieja por apoyar esta iniciativa de investigación y conservación en los municipios de La Primavera y Puerto Carreño, Vichada, cuenca alta y media del río Bita.

\section{Referencias}

Ayala, G., Viscarra, M. E. y Wallace, R. (2010). Density and activity patterns of ocelots (Leopardus pardalis) in Río Hondo, Madidi National Park and Integrated Management Natural Area, La Paz, Bolivia. Revista Boliviana de Ecología y Conservación Ambiental, 28, 119-129.

Blake, J. G., Mosquera, D., Loiselle, B. A., Swing, K., Guerra, J. y Romo, D. (2012). Temporal activity patterns of terrestrial mammals in lowland rainforest of Eastern Ecuador. Ecotropica, 18, 137146.

Bowkett, A., Rovero, F. y Marshall, A. (2007). The use of camera-trap data to model habitat use by antelope species in the Udzungwa Mountain forests, Tanzania. African Journal of Ecology, 46, 479-487.

Cadena, A., Álvarez, J., Sánchez, F., Ariza, C. I., Albesiano, A. (1998). Dieta de los murciélagos frugívoros en la zona árida del río Chicamocha (Santander, Colombia). Boletín de la Sociedad de Biología, 69, 47-53.

Cervantes, F. y Yépez, L. (1995). Species richness of mammals from the vicinity of Salina Cruz, Coastal Oaxaca, México. Anales del Instituto de Biología. Serie Zoología, 66 (1), 113-122.

Colwell, R. K. (2006). EstimateS: Stadistical estimation of species richness and shared species from samples. Version 8. http:// purl.oclc.org/ estimates.

Corporinoquia, HNA Ingeniería y ASODESAM. (2015). Caracterización ambiental y ecosistemas estratégicos en la cuenca del río Bita departamento del Vichada. Bogotá D.C., Colombia: Corpoorinoquia, HNA Ingeniería y ASODESAM. 233 pp.

Correa, H. D., Ruiz, S. L., Arévalo, L. M. (Eds.). (2006). Plan de acción en biodiversidad de la cuenca del Orinoco-Colombia/2005-2015. (Propuesta Técnica). Bogotá, D.C., Colombia: Corporinoquia, Cormacarena, Instituto de Investigación de Recursos Biológicos Alexander von Humboldt, Unitrópico, Fundación Omacha, Fundación Horizonte Verde, Pontificia Universidad Javeriana, UNILLA-NOS, WWF-Colombia, GTZ-Colombia. 330 pp.

Cueva, X. A., Morales, N., Brown, M. y Peck, M. (2010). Macro y mesomamíferos de la Reserva Comunitaria Santa Lucía, Pichincha, Ecuador. Boletín Técnico 9, Serie Zoológica, 6, 98-110.

Díaz-Pulido, A. y Payán-Garrido, E. (2011). Densidad de ocelotes (Leopardus pardalis) en los llanos Colombianos. Mastozoología Neotropical, 18 (1), 63-71.

Emmons, L. H. y Feer, F. (1997). Neotropical rainforest mammals. A field guide. Chicago: The University of Chicago Press. 281 pp.

Emmons, L. H. (1988). A field study of ocelots in Peru. Revue d'Ecologie: La Terre et la Vie, 43, 133157.

Ferrer Pérez, A., Beltrán, M., Díaz-Pulido, A. P., Trujillo, F., Mantilla-Meluk, H., Herrera, O., 
Alfonso, A. F. y Payán, E. (2009). Lista de los mamíferos de la cuenca del río Orinoco. Biota Colombiana, 10, 179-207.

Gaidet-Drapier, N., Frtz, H., Bogarel, M., Renaud, P. C., Poilecot, P. y Chardonne, P. (2006). Cost and efficiency of large mammal census techniques: comparison of methods for a participatory approach in a communal area, Zimbabwe. Biology Conservation, 15, 735-754.

Garrote, G., Rodríguez-Castellanos, P., Trujillo, F. y F. Mosquera-Guerra. (2017). Características de los ataques de jaguar (Panthera onca) sobre el ganado y evaluación económica de las pérdidas en fincas ganaderas de los Llanos Orientales (Vichada, Colombia). En Castaño-Uribe C., Lasso, C. A., Hoogesteijn, R. y Payán-Garrido, E. (Eds.). II. Conflicto entre felinos y humanos en América Latina. Pp. 89-102. Bogotá, D. C., Colombia: Serie editorial Fauna silvestre neotropical. Instituto de Investigación de Recursos Biológicos Alexander von Humboldt (IAvH).

Gompper, M. E., Kays, R. W., Ray, J. C., Lapoint, S. D., Bogan, D. A. y Cryan, J. R. (2006). Comparison of noninvasive techniques to survey carnivore communities in Northeastern North America. Wildlife Society Bulletin, 34, 1142-1151.

Gotelli, N. J. y Colwell, R. K. (2001). Quantifying biodiversity: procedures and pitfalls in the measurement and comparison of species richness. Ecology Letters, 4, 379-391.

Haines, A., Janecka, J., Tewes, M., Grassman, L. Jr. y Morton, P. (2006). The importance of private ands for ocelots Leopardus pardalis in the United States. Oryx, 40, 1-5.

Harmsen, B., Foster, R., Silver, S., Ostro, L. y Doncaster, P. (2010). Differential use of trails by forest mammals and the implications for cameratrap studies: a case study from Belize. Biotropica, 42, 126-133.

Hernández Camacho, J. (1997). Síntesis de los biomas de Colombia. En Seminario Biodiversidad y Manejo de Fauna Silvestre, Palmira (Colombia). Pp: 11-13.
Holden, J. y Neang, T. (2009). Small carnivore records from the Cardamom Mountains, southwestern Cambodia. Small Carnivore Conservation, 40, 16-21.

Kelly, M. J. y Holub, E. L. (2008). Camera trapping of carnivores: Trap success among camera types and across species, and habitat selection by species, on Salt Pond Mountain, Giles County, Virginia. Northeast Naturalist, 15, 249-262.

Krausman, P. (2002). Introduction to wildlife management. Nueva Jersey, EEUU: Prentice Hall. $341 \mathrm{pp}$.

Lira-Torres, I. y Briones-Salas, M. (2012). Impacto de la ganadería extensiva y cacería de subsistencia sobre la abundancia relativa de mamíferos en la Selva Zoque, Oaxaca, México. Therya, 2, 217-244.

Lira-Torres, I., Galindo-Leal, C. y Briones-Salas, M. (2012). Mamíferos de la selva Zoque, México: riqueza, uso y conservación. Revista de Biología Tropical (International Journal of Tropical Biology), 60, 781-797.

Lira-Torres, I. y Briones-Salas, M. (2012). Abundancia relativa y patrones de actividad de los mamíferos de los Chimalapas, Oaxaca, México. Acta Zoológica Mexicana (n. s.), 28(3), 566585.

Maffei, L., Cuellar, E. y Noss, J. (2002). Uso de trampas cámara para la evaluación de mamíferos en el ecotono Chaco-Chiquitanía. Revista boliviana de ecología y conservación ambiental, 11, 55-65.

Maffei, L., Cuellar, E. y Noss, A. (2004). One thousand jaguars (Panthera onca) in Bolivia's Chaco? Camera trapping in the Kaa-Iya National Park. Journal of Zoology, 262, 295-304.

Maffei, L., Noss, A., Cuellar, E. y Rumiz, D. (2005). Ocelot (Felis pardalis) population densities, activity and ranging behavior in the dry forests of eastern Bolivia: data from camera trapping. Journal of Tropical Ecology, 21, 1-6.

Medellín, R., Azuara, D., Maffei, L., Zarza, H., Bárcenas, H., Cruz, E., Legaria, R., Lira, I., Ramos-Fernández, G. y Ávila, S. (2006). Censos 
y Monitoreo. En Chávez, C. y Ceballos, G. (Eds.). El jaguar mexicano en el siglo XXI: situación actual y manejo. Pp: 25-35. México. D. F.: CONABIOALIANZA WWF TELCEL-Universidad Nacional Autónoma de México.

Magurran, A. E. (1988). Ecological diversity and its measurement. New Jersey: Princeton University Press. 179 pp.

Monroy-Vilchis, O., Rodríguez-Soto, C., ZarcoGonzález, M. y Urios, V. (2009). Cougar and jaguar habitat use and activity patterns in Central Mexico. Animal Biology, 59, 145-157.

Monroy-Vilchis, O., Zarco-González, M., Rodríguez-Soto, C., Soria-Díaz, L. y Urios, V. (2011.) Fototrampeo de mamíferos en la Sierra Nanchititla, México. Revista de Biología Tropical (International Journal of Tropical Biology), 59, 373383.

Morales-Jiménez. A. L., Sánchez, F., Poveda, K. y Cadena, A. (2004). Mamíferos Terrestres y Voladores de Colombia. Bogotá, Colombia. 248 pp.

Moreno, C. E. (2001). Métodos para medir la biodiversidad. Zaragoza: M\&T-Manuales y Tesis SEA, vol.1. 84 pp.

Mosquera-Guerra, F., Trujillo, F., MantillaMeluk, H. y Díaz-Pulido, A. (2017). Capítulo 10. Mastofauna. En Trujillo, F y Lasso, C. A. (Eds.). Caracterización biológica de la cuenca del río Bita (Vichada, Colombia). Pp. 297-333. Bogotá, D. C., Colombia: Serie editorial Recursos hidrobiológicos y pesqueros continentales de Colombia. Instituto de Investigación de Recursos Biológicos Alexander von Humboldt (IAvH).

Muñoz-Saba, Y., Trujillo, F., Calvo-Roa, N., Cañón, S. y Mosquera-Guerra, F. (2016). Mamíferos de las cuencas de los ríos Meta y Bita. En Trujillo, F., Antelo, R. y Usma, S. (Eds). Biodiversidad de la cuenca baja y media del río Meta. Pp 248-273. Bogotá, D.C: Fundación Omacha, Fundación Palmarito, WWF.

Naranjo, L.G. y Amaya, J. D. (2009). Plan Nacional de las especies migratorias. Diagnóstico e identificación de acciones para la conservación y el manejo sostenible de las especies migratorias de la biodiversidad en Colombia. Bogotá D.C., Colombia: Ministerio de Ambiente, Vivienda y Desarrollo Territorial, WWF -Colombia. 214 p.

O'Brien, T., Kinnaird, M. y Wibisono, H. (2003). Crouching tigers, hidden prey: Sumatran tiger and prey populations in a tropical landscape. Animal Conservation, 6, 131-139.

Pardo-Martínez, A. y Rangel-Ch, J. O. (2014). Mamíferos de la Orinoquia de Colombia. En Rangel-Ch., J. O. (Ed.). Colombia diversidad biótica XVI. La región de la Orinoquia Colombiana. Pp: 751 784. Bogotá D.C., Colombia: Instituto de Ciencias Naturales, Universidad Nacional de Colombia.

Pina, G. P. L., Gamez, R. A. C. y González, C. A. L. (2004). Distribution, habitat association and activity patterns of medium and large sized mammals of Sonora, Mexico. Natural Areas Journal, 24, 354-357.

Pinto de Sá Alves, L. C. y Andriolo, A. (2005). Camera traps used on the mastofaunal survey of Araras Biological Reserve, IEF-RJ. Revista Brasilera Zootecnia, 2, 231-246.

Ramírez-Chaves, H. E. y Suárez-Castro, A. F. (2014). Adiciones y cambios a la lista de mamíferos de Colombia: 500 especies registradas para el territorio nacional. Mammalogy Notes | Notas Mastozoológicas Sociedad Colombiana de Mastozoología, 1(2), 31-34.

Ramírez-Chaves, H. E., Suárez-Castro, A. F. y González-Maya, J. F. (2016). Cambios recientes a la lista de los mamíferos de Colombia. Mammalogy Notes | Notas Mastozoológicas Sociedad Colombiana de Mastozoología, 3,(1), 1-7.

Roberts, C. W., Pierce, B. L., Braden, A. W., López, R. R., Silvy, N. J., Frank, P. A. y Ranzom, D. (2006). Comparison of camera and road survey estimates for white-tailed deer. Journal Wildlife Manage, 70, 263-267.

Rodríguez-Mahecha, J. V., Alberico, M., Trujillo, F. y Jorgenson, J. (Eds.). (2006). Libro Rojo de los Mamíferos de Colombia. Serie libros rojos de las especies amenazadas de Colombia. Bogotá 
D. C., Colombia: Conservación Internacional Colombia, Ministerio de Ambiente, Vivienda y Desarrollo Territorial. 433 pp.

Romero, M., Galindo, G., Otero, J. y Armenteras, D. (2004). Ecosistemas de la cuenca del Orinoco colombiano (Informe técnico). Bogotá D.C., Colombia: Instituto de Investigación de Recursos Biológicos Alexander von Humboldt. 189 pp.

Rovero, F. y Marshall, A. (2009). Camera trapping photographic rate as an index of density in forest ungulates. Journal Applied Ecology, 46, 1011-1017.

Rowcliffe, J. y Carbone, C. (2008). Surveys using camera traps: are we looking to a brighter future? Animal Conservation Journal, 11, 185-186.

Silveira, L., Ja'como, A. y Diniz-Filhoa, J. (2003). Camera trap, line transect census and track surveys: a comparative evaluation. Biological Conservation, 114, 351-355.

Solari, S., Muñoz-Saba, Y., Rodríguez-Mahecha, J. V., Defler, T., Ramírez-Chaves, H. E. y Trujillo, F. (2013). Riqueza, endemismo y conservación de los mamíferos de Colombia. Mastozoología Neotropical, 20 (2), 301-365.

Srbek-Araujo, A. C. y García, A. (2005). Is camera trapping an efficient method for surveying mammals in Neo- tropical forests? A case study in south-eastern Brazil. Journal of Tropical Ecology, $21121-125$.

Sutherland, W. (1996). Ecological Census Techniques. Cambridge, Gran Bretaña: Cambridge University. 336 pp.

Swan, M., Di Stefano, J., Chistie, F., Steel, E. y York, A. (2014]). Detecting mammals in heterogeneous landscapes: implications for biodiversity monitoring and management. Biodiversity and Conservation, 23, 343-355.

Tobler, M., Carrillo-Percastegui, S., Leite, R., Mares, R. y Powell, G. (2008). An evaluation of camera traps for inventorying large- and medium-sized terrestrial rainforest mammals. Animal Conservation Journal, 11, 169-178.
Tobler, M.W., Carrillo-Percastegui, S. E. y Powell, G. (2009). Habitat use, activity patterns and use of mineral licks by five species of ungulate in south-eastern Peru. Journal of Tropical Ecology, 25, 261-270.

Trolle, M. y Kéry, M. (2003). Estimation of ocelot density in the Pantanal using capture-recapture analysis of camera-trapping data. Journal of Mammalogy, 84, 607-614.

Trolle, M. (2008). Brazilian tapir density in the Pantanal: a comparison of systematic cameratrapping and linetransect surveys. Biotropica, 40,211-217.

Trujillo, F. y Mosquera, F. (2016). Caracterización, uso y manejo de la mastofauna asociada a los morichales de los Llanos Orientales colombianos. Capítulo 7. En Lasso, C. A., Colonnello, G., y Moraes, R, M. (Eds). XIV. Morichales, cananguchales y otros palmares inundables de Suramérica. Parte II: Colombia, Venezuela, Brasil, Perú, Bolivia, Paraguay, Uruguay y Argentina. Pp. 191-219. Serie editorial Recursos hidrobiológicos y pesqueros continentales de Colombia. Bogotá, D. C., Colombia: Instituto de Investigación de Recursos Biológicos Alexander von Humboldt $(\mathrm{IAvH})$.

UICN-Unión Internacional para la Conservación de la Naturaleza. (2016). Recuperado de: https:/ / www.iucn.org/es

Van Schaik, C. P. y Griffiths, M. (1996). Activity Periods of Indonesian Rain Forest Mammals. Biotropica, 28, 105-112.

Walker, S., Novaro, A. y Nichols, J. (2000). Consideraciones para la estimación de abundancia de poblaciones de mamíferos. Mastozoología Neotropical, 7, 73-80.

Weckel, M., Giuliano, W. y Silver, S. (2006). Jaguar (Panthera onca) feeding ecology: distribution of predator and prey through time and space. Journal of Zoology, 270, 25-30.

Wilson, D. E., Rusell, F., Nichols, J. D., Rudran, R. y Foster, M. S. (Eds.). (1996). Measuring 
and Monitoring Biological Diversity, Standard Methods for Mammals. Washington and London: Smithsonian Institution Press. 409 pp.

Wilson, D. E. y Reeder, D. A. M. (Eds.). (2005). Mammal Species of the World. A Taxonomic and Geographic Reference. Third Edition. Baltimore: The Johns Hopkins University Press. 142 pp.
Whittaker, R. H. (1972). Evolution and measurement of species diversity. Taxon, 21, (2/3), 213-251.

Yasuda, M. (2004). Monitoring diversity and abundance of mammals with camera traps: a case study on Mount Tsukuba, central Japan. Mammal Study, 29, 37-46.

Zar, J. H. (1999). Biostatistical analysis. Tercera edición. New Jersey: Prentice Hall. 988 pp.

Anexo 1. Mamíferos medianos y grandes registrados en la cuenca alta y media del río Bita, Vichada, Colombia.

Disponible en línea: http://revistas.humboldt.org.co/index.php/biota/rt/suppFiles/578/0

\section{Federico Mosquera-Guerra}

Fundación Omacha

Universidad Nacional de Colombia, Laboratorio de Ecología del Paisaje y Modelación de Ecosistemas

Bogotá, Colombia

federico.mosqueraguerra@gmail.com

Fernando Trujillo

Fundación Omacha

Bogotá, Colombia

fernando@omacha.org

Angélica P. Diaz-Pulido

Instituto de Investigación de Recursos Biológicos

Alexander von Humboldt

Villa de Leyva, Colombia

adiaz@humboldt.org.co

Hugo Mantilla-Meluk

Universidad del Quindío

Armenia, Colombia

hugo.mantillameluk@gmail.com
Diversidad, abundancia relativa y patrones de actividad de los mamíferos medianos y grandes, asociados a los bosques riparios del río Bita, Vichada, Colombia

Citación del artículo: Mosquera-Guerra, F., Trujillo, F., Diaz-Pulido, A. P. y Mantilla-Meluk, H. (2018). Diversidad, abundancia relativa y patrones de actividad de los mamíferos medianos y grandes, asociados a los bosques riparios del río Bita, Vichada, Colombia. Biota Colombiana, 19(1), 202-218. DOI: 10.21068/c2018v19n01a13.

Recibido: 20 de abril de 2017

Aprobado: 20 de noviembre de 2017 